
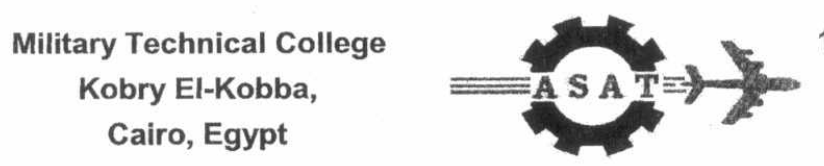

11-th International Conference

on Aerospace Sciences \&

Aviation Technology

\title{
ANALYSIS OF TORSION ACTUATION IN ACTIVE THIN- WALLED SINGLE-CELL COMPOSITE BEAMS
}

\author{
Moustafa Kinawy", Wagdy E. Abdel-Ghany"
}

\begin{abstract}
A modified approach is developed to predict the deformation of active thin-walled single-cell active composite beam of general cross-section under general loading. The theory takes into consideration the transverse shear due to restrained warping, and the bending wall effect. The analytical model for the equations of motions consisting of six non-homogenous coupled differential equations of $14^{\text {th }}$ order is used. Then a study is made on torsion actuation of beams. Then simplification is made to study the efficiency of possible types of torsion actuation techniques. Finally rectangular active box-beams are studied under three different torsion actuation techniques.
\end{abstract}

\section{KEYWORDS}

Smart structures - Composite beams - Active materials - Torsion Actuation

\section{NOMENCLATURE}

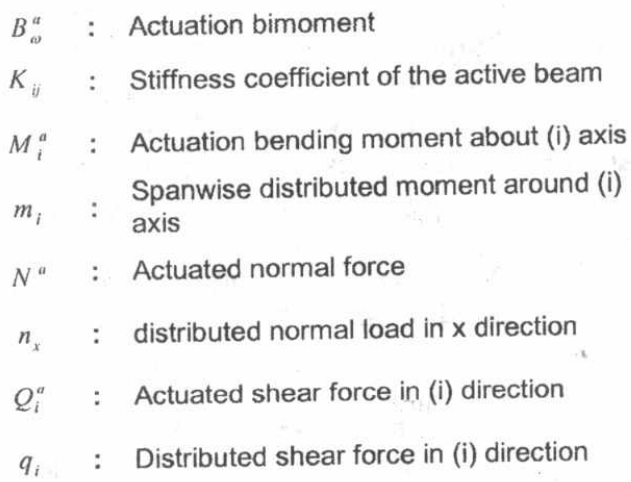

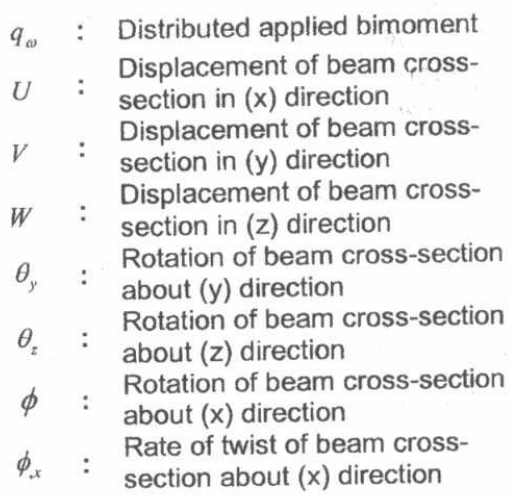
$\phi_{x} \quad$ : section about $(\mathrm{x})$ direction

* Research Engineer, Aerospace Research Center, , Cairo, Egypt, moskinl@yahoo.com

** Research Engineer, Aerospace Research Production AinShams University, Cairo, Egypt 


\section{INTRODUCTION}

Thin-walled construction has been widely used in the aerospace industry. Many primary structural components such as aircraft wing spars and the helicopter rotor blades now feature composite thin-walled designs. These composite designs are light weight and offer innovative characteristics. The inherit tailorability is being investigated to enhance these structures. Specifically, the elastic coupling between several deformations are typically not exploited in composite designs. These couplings arise due to the anisotropic or directional nature of fibrous composites. One of the most dramatic applications of this technology is used to prevent divergence of swept-forward wings. By embedding active material within the parent composite layers, we can acquire active controlled structure. Successful applications of active thin-walled structures require the development of new analytical tools, which are both sufficiently accurate and computationally efficient. Recent efforts toward the creation of new analytical tool for composite beams have led to the development of several finite element based methods. The finite element models provide varying degrees of analytical flexibility depending on the level of computational level required. Direct analytical models have been developed, dealing with the beams as cylindrical structures. Most of these models neglect the bending effect of walls under deformation.

A systematic approach is taken to develop both a qualitative and quantitative understanding of composite box-beam behavior. Special consideration is given to modeling the effect of anisotropy elasticity, laminated construction, bending of
walls.

Esmat [1] derived a mathematical model for static response of multicell composite beams. Chopra et. Al. [2] studied symmetric and antisymmetric layup graphite epoxy box-beams of closed cross-section. Their analytical predictions underestimate bending-induced twist. Ehlers [3] studied the ability to deform a structure through the use of coupling with out-of -plane deformation modes, and the use of warping torsion coupling permits axially aligned actuators to control twist of a closed cell section. Libove [4] introduced a theory for computing shear flows, cross-sectional normal stresses, and rate of twist in linearly elastic singlecell thin-walled beams with anisotropic walls. Chaaban et. AL [5] introduced a mathematical model for cylindrical beams made of anisotropic materials, including the effect of active layers. The model introduces the effect of bending of walls. Then they applied this model on symmetric composite box-beams to evaluate their behavior under different bending actuation techniques. The equations of motion of the beam consist of a system of six non-homogenous ordinary differential equations of the $14^{\text {th }}$ order.

The present work uses their model, and applies it on box-beams under different torsion actuation techniques. The beams are embedded with active layers within their layers. Also the layup of the beams allows the different actuation techniques by using elastic coupling between deformations.

Many of the current direct analytical methods for composite thin-walled beams are based heavily on thin-walled beam theory. The model was developed in accordance with this theory also, but taking into consideration the bending of walls. Some assumptions were considered regarding the geometry and materials of the beam. The equations of motion of the beam are developed under different 
loading. Then applications are made on a rectangular-shaped box-beam under direct twist, warping-torsion coupling, and extension-torsion coupling.

The objectives of this study are:

1. To validate and evaluate an existing mathematical model of the beam.

2. To use the developed model to evaluate different torsion actuation techniques of single-cell thin-walled composite active beam.

\section{ACTUATED BEAM DESCRIPTION AND ASSUMPTIONS}

By the actuated beam, it is meant a one dimensional structure with thin-walls and a general shaped single cell cross-section. The thickness of the thin walls can vary arbitrarily around the cell. The walls are formed of composite laminates of different ply orientations and ply materials. Each ply material can be either active materials or non-active material (passive) fiber composites.

Two coordinate systems are associated to a generic cross-section of the actuated single cell composite beam as in Fig. 1 :

- Global Cartesian coordinate system $(x, y, z)$ situated at the root of the beam and it is fixed in space.

- Local Cartesian coordinate system $(x, t, n)$ located at the mid-plane of the laminate forming the beam cross-section, and it moves around the contour of the section with a circumferential coordinate (s).

In the local coordinate system, ( $t$ ) is the tangential coordinate and $(n)$ is the outward normal coordinate to the mid-plane contour of the beam wall. In both coordinate systems the $x$-axis is coinciding and in the direction of the axis of the beam.

The model for the thin-walled, anisotropically actuated single-cell, composite beam is based on the following assumptions

1- The shape of the cross-section is maintained during deformation but out-of-plane displacements are allowed.

2- The wall thickness is small compared to the other dimensions so that the problem can be treated as a thin-wall plane stress problem.

3- The transverse in-plane normal stresses are negligible (i.e. no internal pressure).

4- The rate of twist can vary along the length of the beam and it is a measure of the warping due to torsion of the cross-section.

5- The active material sections can be modeled with linear piezoelectric constitutive relations and are excited with an electric field applied through the thickness of the actuator.

The equations of motion of the beam are six differential equations:

- 3 differential equations from forces equilibrium

- 3 differential equations from moment equilibrium

The derivation of these equations is in [5]. 


\section{Forces equilibrium}

$$
\begin{gathered}
K_{11} \frac{d^{2} U}{d x^{2}}+K_{12} \frac{d^{2} V}{d x^{2}}-K_{12} \frac{d^{2} \theta_{z}}{d x^{2}}+K_{13} \frac{d^{2} W}{d x^{2}}+K_{13} \frac{d \theta_{y}}{d x}+K_{11} \frac{d^{3} \phi}{d x^{3}} \\
+K_{14} \frac{d^{2} \phi}{d x^{2}}+K_{15} \frac{d^{2} \theta_{y}}{d x^{2}}+K_{16} \frac{d^{2} \theta_{z}}{d x}=\frac{d N^{a}}{d x}-n_{x} \\
K_{21} \frac{d^{2} U}{d x^{2}}+K_{22} \frac{d^{2} V}{d x^{2}}+K_{23} \frac{d^{2} W}{d x^{2}}+K_{27} \frac{d^{3} \phi}{d x^{3}}+K_{24} \frac{d^{2} \phi}{d x^{2}}+K_{25} \frac{d^{2} \theta_{y}}{d x^{2}} \\
+K_{26} \frac{d^{2} \theta_{z}}{d x}+K_{23} \frac{d \theta_{y}}{d x}-K_{22} \frac{d \theta_{z}}{d x}=\frac{d Q_{y}^{a}}{d x}-q_{y} \\
K_{31} \frac{d^{2} U}{d x^{2}}+K_{32} \frac{d^{2} V}{d x^{2}}+K_{33} \frac{d^{2} W}{d x^{2}}+K_{37} \frac{d^{3} \phi}{d x^{3}}+K_{34} \frac{d^{2} \phi}{d x^{2}}+K_{35} \frac{d^{2} \theta_{y}}{d x^{2}} \\
+K_{36} \frac{d^{2} \theta_{z}}{d x}+K_{33} \frac{d \theta_{y}}{d x}-K_{32} \frac{d \theta_{z}}{d x}=\frac{d Q_{z}^{a}}{d x}-q_{z}
\end{gathered}
$$

\section{Moment equilibrium}

$$
\begin{aligned}
& K_{51} \frac{d^{2} U}{d x^{2}}+K_{52} \frac{d^{2} V}{d x^{2}}+K_{53} \frac{d^{2} W}{d x^{2}}-K_{13} \frac{d U}{d x}-K_{23} \frac{d V}{d x}-K_{33} \frac{d W}{d x}+K_{57} \frac{d^{3} \phi}{d x^{3}}+\left(K_{45}-K_{37}\right) \frac{d^{2} \phi}{d x^{2}} \\
&+K_{55} \frac{d^{2} \theta_{y}}{d x^{2}}+K_{56} \frac{d^{2} \theta_{z}}{d x}-K_{34} \frac{d \phi}{d x}-\left(K_{25}+K_{36}\right) \frac{d \theta_{z}}{d x}-K_{33} \theta_{y}+K_{23} \theta_{z}=\frac{d M_{y}^{a}}{d x}-Q_{z}^{a}-m_{y} \\
& K_{16} \frac{d^{2} U}{d x^{2}}+K_{26} \frac{d^{2} V}{d x^{2}}+K_{36} \frac{d^{2} W}{d x^{2}}-K_{12} \frac{d U}{d x}-K_{22} \frac{d V}{d x}-K_{23} \frac{d W}{d x}+K_{67} \frac{d^{3} \phi}{d x^{3}}+\left(K_{46}+K_{27}\right) \frac{d^{2} \phi}{d x^{2}} \\
&+K_{56} \frac{d^{2} \theta_{y}}{d x^{2}}+K_{66} \frac{d^{2} \theta_{z}}{d x}+K_{24} \frac{d \phi}{d x}+\left(K_{36}+K_{25}\right) \frac{d \theta_{y}}{d x}+K_{23} \theta_{y}+K_{22} \theta_{z}=\frac{d M_{z}^{a}}{d x}-Q_{y}^{a}-m_{z} \\
&-K_{77} \frac{d^{4} \phi}{d x^{4}}-K_{17} \frac{d^{3} U}{d x^{3}}-K_{27} \frac{d^{3} V}{d x^{3}}-K_{37} \frac{d^{3} W}{d x^{3}}+K_{57} \frac{d^{3} \theta_{y}}{d x^{3}}-K_{67} \frac{d^{3} \theta_{z}}{d x^{3}}+K_{14} \frac{d^{2} U}{d x^{2}}+K_{24} \frac{d^{2} V}{d x^{2}} \\
&+K_{34} \frac{d^{2} W}{d x^{2}}+K_{44} \frac{d^{2} \phi}{d x^{2}}+\left(K_{45}-K_{37}\right) \frac{d^{2} \theta_{y}}{d x^{2}}+\left(K_{46}+K_{27}\right) \frac{d^{2} \theta_{z}}{d x^{2}} \\
&+K_{34} \frac{d \theta_{y}}{d x}-K_{24} \frac{d \theta_{z}}{d x}=\frac{d M_{x}^{a}}{d x}-\frac{d^{2} B_{w}^{a}}{d x^{2}}-m_{x}+\frac{d q_{w}}{d x}
\end{aligned}
$$

They represent a coupled system of six linear ordinary non-homogenous differential equations with constant coefficients. Their analytical solution is generally difficult, but by relaxing some of the coupling effects, solutions can be obtained. This can be done by beam tailoring and application of special actuation techniques. In the present work, torsion actuation on box-beam structure is considered.

\section{TORSION ACTUATION}

The torsion deformation of a single-cell box-beam structure can be controlled by several actuation techniques. In the following, three methods are considered;

1. Direct Twist Actuation

2. Extension Actuation with an Extension-Twist coupled Beam

3. Warping Actuation of the beam 
The active beam consists of parent structure made of composite materials, and active material. The parent structure is tailored to remove all unnecessary coupling effects and retain the necessary coupling to transform the different types of induced loading to gain the required torsion actuation. The active material is distributed in order that to be able to induce the required load.

\section{Direct Twist Actuation}

The direct twist actuation can be done either by:

- Shear Deformation active material

or

- Isotropic active material

Although some materials do exhibit shear strain-electric field coupling, they are not in the forms useful for thin-walled structures. There are currently known three approaches to achieve induced strain anisotropy:

1. orthotropic piezoelectric materials

2. specially attached isotropic piezoelectric

3. unidirectional piezoelectric fiber composites

In case of torsion actuation of single-cell composite beam structure (see Fig. 2), the previous system of equations is reduced to the following:

$$
\frac{d^{4} \phi}{d x^{4}}-\lambda^{2} \frac{d^{2} \phi}{d x^{2}}=\frac{m_{x}^{a}}{K_{77}} \quad ; \quad m_{x}^{a}=\frac{1}{K_{77}} \cdot \frac{d M^{a}}{d x} \quad ; \quad \lambda^{2}=\frac{K_{44}}{K_{77}}
$$

The solution of this system is in the form:

$$
\phi=-\frac{m_{x}^{a} L}{K_{44}}\left(\tanh \lambda L+\frac{1}{\lambda L \cosh \lambda L}\right)\left(\frac{\cosh \lambda x-1}{\lambda}\right)+\left(x-\frac{\sinh \lambda x}{\lambda}-\frac{x^{2}}{2 L}\right)
$$

The average twist angle is

$$
\phi_{a v}=\frac{m_{x}^{a} L}{K_{44}}\left(\frac{\tanh \lambda L}{\lambda}+\frac{1}{\lambda^{2} L \cosh \lambda L}\right)\left(\frac{\sinh \lambda L}{\lambda L}-1\right)+\left(\frac{L}{3}-\frac{\cosh \lambda L-1}{\lambda^{2} L}\right)
$$

The direct twist actuation is performed by inducing shear in the cell walls; i.e. by using anisotropic active material, in the form of Unidirectional Piezoelectric Fiber Composites (UNIPFC).

\section{Extension-Twist Coupling Actuation}

The extension actuation is based on the tailoring of the active single-cell composite beam Structure such that there exist a coupling between extension and torsional 
deformation i.e. $K_{14} \neq 0$. Anisotropic parent structure permits twist actuation through the use of extension-twist coupling where, the extension is actuated through the flanges (actuators) as in Fig.3. In case of Extension-Twist actuation of box beam structure, the previous system of equations is reduced to the following:

$$
\frac{d^{4} \phi}{d x^{4}}-\lambda^{2} \frac{d^{2} \phi}{d x^{2}}=\frac{-K_{14}}{K_{11} K_{77}} \cdot p_{x} \quad ; \quad p_{x}=\frac{d N^{a}}{d x} \quad ; \quad \lambda^{2}=\frac{K_{11} K_{44}+K_{14}^{2}}{K_{11} K_{77}}
$$

After solving the differential equation and applying the boundary conditions we get the following solution:

$$
\phi=\frac{P_{x} L}{\frac{K_{11} K_{44}}{K_{14}}+K_{14}^{2}}\left(\tanh \lambda L+\frac{1}{\lambda L \cosh \lambda L}\right)\left(\frac{\cosh \lambda x}{\lambda}\right)+\left(x-\frac{\sinh \lambda x}{\lambda}-\frac{x^{2}}{2 L}\right)
$$

and The average twist angle is

$$
\phi_{a v}=\frac{P_{x} L}{\frac{K_{11} K_{44}}{K_{14}}+K_{14}}\left(\left(\frac{\sinh \lambda L}{\lambda L}-1\right) \cdot\left(\frac{\tanh \lambda L}{\lambda}+\frac{\sec h \lambda L}{\lambda^{2} L}\right)\right)+\left(\frac{L}{3}-\frac{\cosh \lambda L-1}{\lambda^{2} L}\right)
$$

\section{Warping-Twist Actuation}

The active single-cell composite beam structure will be exactly as the case of extension actuation with four flanges. The warping deformations is created by expanding the upper left and lower right flanges (actuators) and contracting the lower left and upper right actuators as shown in Fig. 4. The resulting out-of-plane deformation simulated the section-warping mode.

This scheme of forces introduces warping at the end of the beam. The torsional deformations are concentrated near the free end in a decay length that is function of the ratio of the torsional to warping stiffness of the beam section, i.e. $K_{44} / K_{77}$.

The internal shear flows that result from warping of the cross-section tend to distort its shape, but the present work assumes rigid diaphragm structure. The active singlecell composite beam model is based on a method that assumes all extensional stiffness is lumped at the flanges with the skins and webs acting only in shear. The active single-cell composite beam in this case will twist due to the applied warping load which is a bimoment arising from the four axial forces in the spar flanges from the active materials. The torsional differential Equation defining this case is that given by Equation (13) with no applied torque. Due to the effect of decay length, it is important to investigate three different cases of boundary conditions for the boxbeam structure:

1. A cantilever constrained from warping at the root and free at its tip.

2. A beam that is constrained from rotation at the root and free to warp at both ends.

3. A beam that is free to warp at both ends and constrained from rotation at both ends. 
Assuming constant section geometry and uniform material distribution along the boxbeam structure, Equation (6) becomes a homogenous linear differential with constant coefficients. Its general solution can be written in the form:

$$
\frac{d^{4} \phi}{d x^{4}}-\lambda^{2} \frac{d^{2} \phi}{d x^{2}}=0 \quad ; \quad \lambda^{2}=\frac{K_{44}}{K_{77}}
$$

After applying the proper boundary conditions for each case, the following solutions are in the following form.

\section{Beam 1:}

$$
\begin{array}{ll}
\text { B.C. } x & =0 ; \quad \phi=0 ; \quad \frac{d \phi}{d x}=0 \\
x & =L ; \quad \frac{d^{2} \phi}{d x^{2}}=\frac{B_{\omega}}{K_{77}} ; \quad \frac{d^{3} \phi}{d x^{3}}=\lambda^{2} \frac{d \phi}{d x} \\
\phi & =\frac{B_{\omega}}{K_{77}}\left(\frac{1}{\lambda L \cosh \lambda L}\right)
\end{array}
$$

the average twist angle is

$$
\phi_{a v}=\frac{B_{\omega}}{K_{77}} \cdot\left(\frac{\sinh \lambda L-\lambda L}{\lambda L \cosh \lambda L}\right)
$$

Beam 2:

$$
\begin{array}{lll}
\text { B.C. } x=0 ; \quad \phi=0 ; & \frac{d^{2} \phi}{d x^{2}}=0 \\
x=L ; \quad & \frac{d^{2} \phi}{d x^{2}}=\frac{B_{\omega}}{K_{77}} ; & \frac{d^{3} \phi}{d x^{3}}=\lambda^{2} \frac{d \phi}{d x} \\
\phi=\frac{B_{\omega}}{K_{77}}\left(\frac{\sinh \lambda x}{\sinh \lambda L}\right) & &
\end{array}
$$

the average twist angle is

$$
\phi_{a v}=\frac{B_{w}}{K_{77}} \cdot\left(\frac{\cosh \lambda L-1}{\lambda L \sinh \lambda L}\right)
$$

\section{Beam 3}

$$
\begin{array}{lll}
\text { B.C. } x=0 ; & \phi=0 ; \quad \frac{d^{2} \phi}{d x^{2}}=0 \\
x=L ; & \frac{d^{2} \phi}{d x^{2}}=\frac{B_{\omega}}{K_{77}} ; \quad \phi=0
\end{array}
$$




$$
\phi=\frac{B_{\omega}}{K_{44}}\left(\frac{\sinh \lambda x}{\sinh \lambda L}-\frac{x}{L}\right)
$$

the average twist angle is

$$
\phi_{a v}=\frac{B_{\omega}}{K_{44} L} \cdot\left(\frac{\cosh \lambda L-1}{\lambda \sinh \lambda L}-\frac{L^{2}}{2 L}\right)
$$

\section{Composite Box-Beam Structure Description}

In the case of direct twist actuation, the beam section is rectangular. It is made from different laminate types. The laminates are made mainly from orthotropic composite layers. The box-beam structure has no flanges as in Fig. 2, and the upper and lower skins are made from two layers of (UNIPFC) at (45) degree to form: laminate $\left( \pm 45^{\circ}\right)$ PFC (PZT/Epoxy). The cross-section of the beam is shown in Fig.5. This configuration permits the Piezoelectric Fiber Composites serves as the active material and structural material without the need for additional materials to carry and distribute loads. The Piezoelectric Fiber Composite (PFC) is an anisotropic actuation material which has the capability of inducing direct, planar, distributed shear stresses in a structure. The PFC actuators consist of piezoelectric fibers which are embedded in a soft epoxy matrix. This composite active layer is sandwiched between two electrode layers to supply the necessary through-thickness applied electrical field. The main advantages of PFC actuators are the tailorability, conformability and improved reliability. It is also easily integrated with parent composite material using manufacturing techniques widely used in aerospace environment. The mechanical properties of the PFC are given in the Table (1).

In the case of extension-twist coupling, conventional isotropic active materials are aligned longitudinally at the places of the four flanges with an anisotropic parent structure will permit twist actuation through the use of extension-twist coupling $\left(K_{14} \neq 0\right)$. The upper and lower skins will be made of two layers of unidirectional Glass/epoxy tape oriented at $45^{\circ}$ with respect to the local $x$ - axis. The beam is shown in Fig.3, and the cross-section of the beam is as in Fig.6. By applying extensional forces at the four flanges from piezoelectric material, a case of twisting will exist due to the extension of the composite box beam structure.

For the case of warping torsion coupling, the upper and lower skins consist of two layers of zero degrees Glass/epoxy unidirectional tape. The actuators are of isotropic piezoelectric material of $1.25 \mathrm{~cm}$ wide and $0.02 \mathrm{~cm}$ thick. They are located in the place of the four flanges as shown in Fig.4. The cross-section of the beam is as in Fig.6. Warping forces are generated through a self-equilibrated load system of four axial forces in flanges. This is accomplished by expanding the upper left and lower right actuators and contracting the lower left and upper right actuators. The resulting self-equilibrating load is a bimoment which causes warping of the section and consequently twist due to the warping-torsion coupling. 
The dimensions of the rectangular cross-section are as follows:

$$
\begin{array}{lll}
\text { Width } & b=50 & (\mathrm{~mm}) \\
\text { Depth } & a=6.25 & (\mathrm{~mm})
\end{array}
$$

and the mechanical properties of the parent composite materials and the piezoelectric actuators are given in Table 1.

\section{RESULTS}

The method is done by first: computing the properties for each laminate of the beam. Then calculating the laminate stiffness coefficients. Then the active beam stiffness coefficients $K_{i j}$ are calculated by evaluating the corresponding contour integration. Finally the twist angle distribution of each case is calculated. The three cases of actuation are shown in the next three Figures. All of the three structures have the same effective torsional rigidity defined as the ratio of the applied torque to the twist rate with all other forces and applied field set to zero. For all torsion actuation methods, the twist distribution per unit length along the structure per unit strain $\left(\frac{\phi_{a v}}{m_{x} L}, \frac{\phi_{a v}}{p_{x} L}, \frac{\phi_{a v}}{B_{\omega} L}\right)$ are shown in Fig. 7,8 and 9.

\section{Parametric Analysis}

Based on the results the most effective torsion actuation method is the Direct Twist by the cell wall shear actuation technique and the Extension-Twist actuation. Both have a considerable effect on the twist angle distribution along the whole length of the structure. Whereas the Warping-Twist actuation has only localized effect on a small portion along the length of the beam structure. For the three cases we'll make a parametric analysis to study the most effective parameters of the structure geometric and material characteristics. Changing the aspect ratio $(\mathrm{b} / \mathrm{a})$, the web plies number, and beam length are changed as in Tables 1, 2, and 3 respectively.

From the results in Tables, we see that as the aspect ratio increases the average twist angle increases. This was expecting because decreasing the aspect ratio the torsion stiffness increases.

\section{CONCLUSION}

The present work uses a mathematical model for active thin-walled composite single-cell beams of general cross-section, taking into account the effect of wall bending. Three torsion actuation techniques are studied on composite box-beam by using the deformation control of the structure. This is done by tailoring the parent structure to allow direct twist actuation, extension-twist coupling, and warping-twist coupling.

The results show both cases of direct twist, and extension-twist coupling have a widespread effect along the beam length. Whereas in warping actuation (Beam 1, 
and Beam 2) there is only local effect of twist near the end tip of the beam. With introducing the new boundary condition (Beam 3 ) the twist is apparent along the beam length (i.e. amplification in twist). This beam case distribution is affected mostly by changing the effective length $(\lambda)$, which is $\frac{K_{44}}{K_{77}}$ (the length of the beam which has apparent effect of twist). By introducing a figure of merit $\frac{\phi_{a v}}{F L}$ (the average twist per unit axial load per unit length), we can compare between the effectiveness of the last two actuation techniques. It shows that the extensiontwist actuation is greater than the warping (Beam 1,2) actuation by the factor of 2.28 .

\section{APPENDIX}

The stiffness coefficients in the equilibrium equations (28-33) are defined as follows:

$$
\begin{aligned}
& K_{11}=\oint L_{11} d s \\
& K_{12}=\oint L_{12} Y_{, s} d s \quad ; \quad K_{13}=\oint L_{12} Z_{, s} d s \\
& K_{14}=\oint L_{12}\left(\frac{-2 A \alpha}{\Gamma}\right) d s \quad ; \\
& K_{15}=\oint\left(L_{11} Z+L_{13} Y_{s}\right) d s \\
& K_{16}=\oint\left(-L_{11} Y+L_{13} Z_{s}\right) d s \text {; } \\
& K_{17}=\oint L_{11} \Psi(s) d s \quad ; \quad K_{22}=\oint L_{22}\left(Y_{s}\right)^{2} d s \\
& K_{23}=\oint L_{22} Y_{s} Z_{s} d s \quad \text {; } \\
& K_{24}=\oint L_{22}\left(\frac{-2 A \alpha}{\Gamma}\right) Y_{s} d s \\
& K_{25}=\oint\left(L_{12} Z+L_{23} Y_{s}\right) Y_{, s} d s ; \quad K_{26}=\oint\left(-L_{12} Y+L_{23} Z_{s}\right) Y_{, s} d s \\
& K_{27}=\oint L_{12} \Psi(s) Y_{s,} d s \quad ; \quad K_{33}=\oint L_{22}\left(Z_{s}\right)^{2} d s \quad ; \quad K_{34}=\oint L_{22}\left(\frac{-2 A \alpha}{\Gamma}\right) Z_{s} d s \\
& K_{35}=\oint\left(L_{12} Z+L_{23} Y_{, s}\right) Z_{, s} d s ; K_{36}=\oint\left(-L_{12} Y+L_{23} Z_{, s}\right) Z_{, s} d s \\
& K_{37}=\oint L_{12} \Psi(s) Z_{s} d s ; \quad K_{44}=\oint L_{22}\left(\frac{-2 A \alpha}{\Gamma}\right) d s \\
& K_{45}=\oint\left(L_{12} Z+L_{23} Y_{s}\right)\left(\frac{-2 A \alpha}{\Gamma}\right) d s ; \quad K_{46}=\oint\left(-L_{12} Y+L_{23} Z_{s}\right)\left(\frac{-2 A \alpha}{\Gamma}\right) d s \\
& K_{47}=\oint L_{12} \Psi(s)\left(\frac{-2 A \alpha}{\Gamma}\right) d s \quad ; \quad K_{55}=\oint\left(L_{11} Z+L_{13} Y_{s}\right) Z+\left(L_{13} Z+L_{33} Y_{s s}\right) Y_{s} d s \\
& K_{56}=\oint\left(-L_{11} Y+L_{13} Z_{s}\right) Z+\left(-L_{13} Y+L_{33} Z_{s, s}\right) Y_{s} d s \quad ; \quad K_{57}=\oint\left(L_{11} Z+L_{13} Y, s\right) \Psi(s) d s \\
& K_{66}=\oint\left(-L_{11} Y+L_{13} Z_{s}\right)(-Y)+\left(-L_{13} Y+L_{33} Z_{s}\right) Z_{s,} d s ; \quad K_{67}=\oint\left(L_{11}(-Y)+L_{13} Z_{s}\right) \Psi(s) d s \\
& K_{77}=\oint L_{11} \Psi^{2}(s) d s
\end{aligned}
$$

where $\Gamma$ is the contour length. 
FIGURES AND TABLES

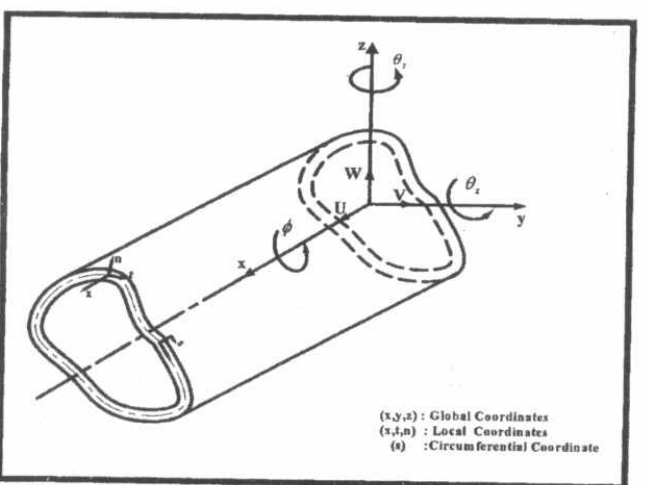

Fig. 1-cylindrical single cell beam

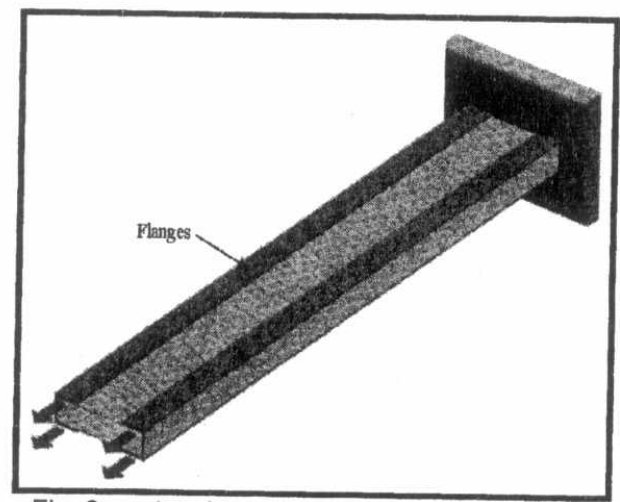

Fig. 3 - extension-twist actuation of box beam structure

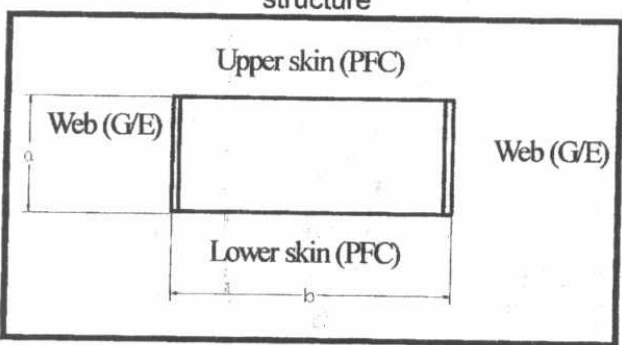

Fig. 5-beam section for direct twist application

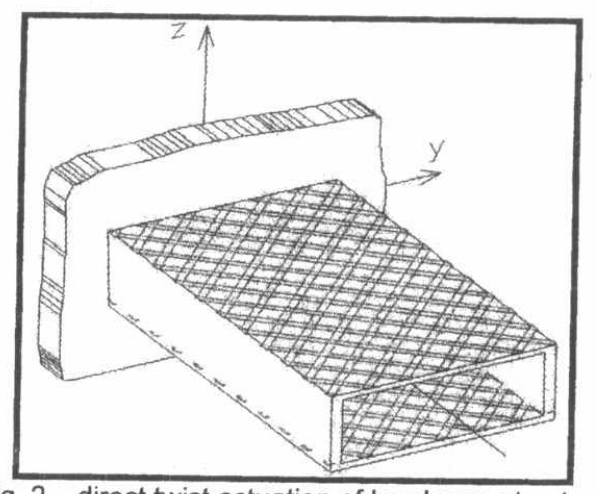

Fig. 2 - direct twist actuation of box beam structure

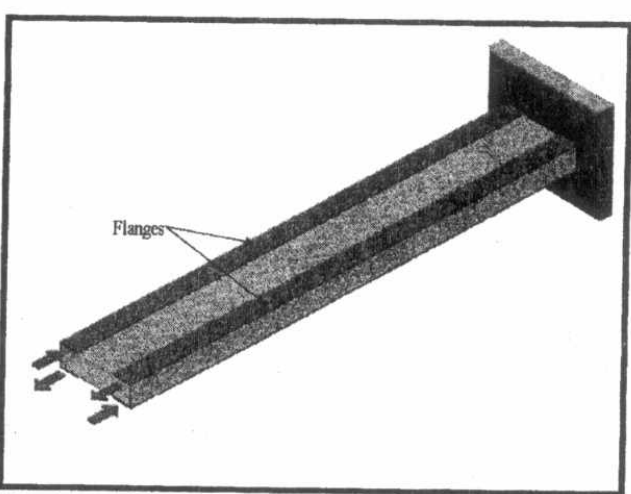

Fig. 4-warping-twist actuation of box beam structure

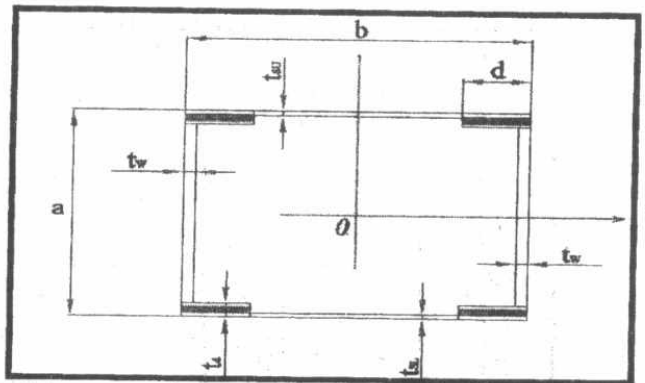

Fig. 6-beam section for extension-actuation coupling, and warping actuation 


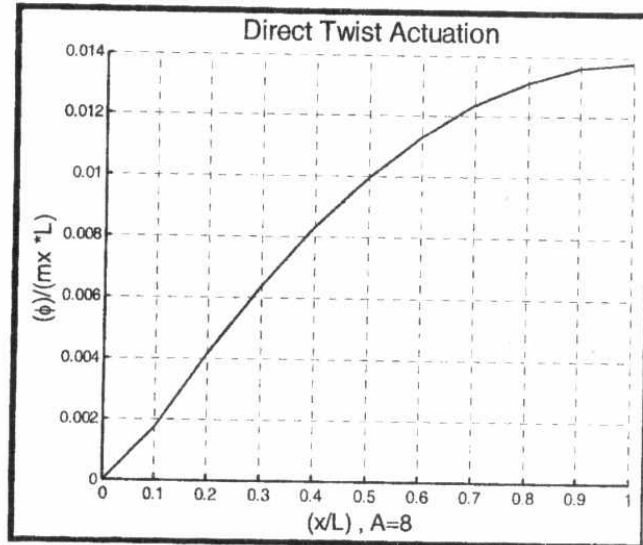

Fig. -7 twist angle distribution for direct twist actuation

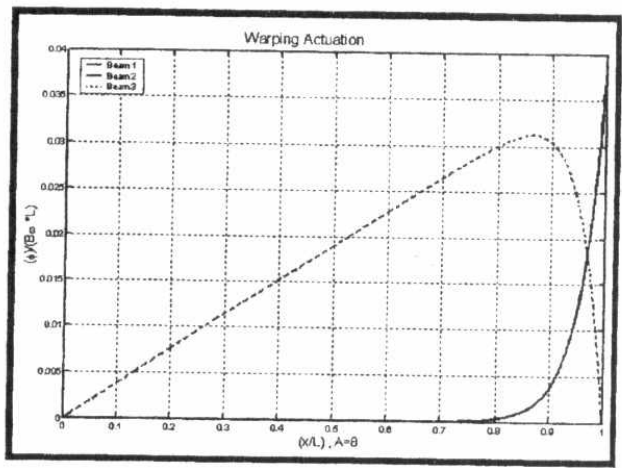

Fig.- 9 twist angle distribution for warping-twist actuation for beam $1,2,3$

Note: Beam 1, and 2 are almost coincident

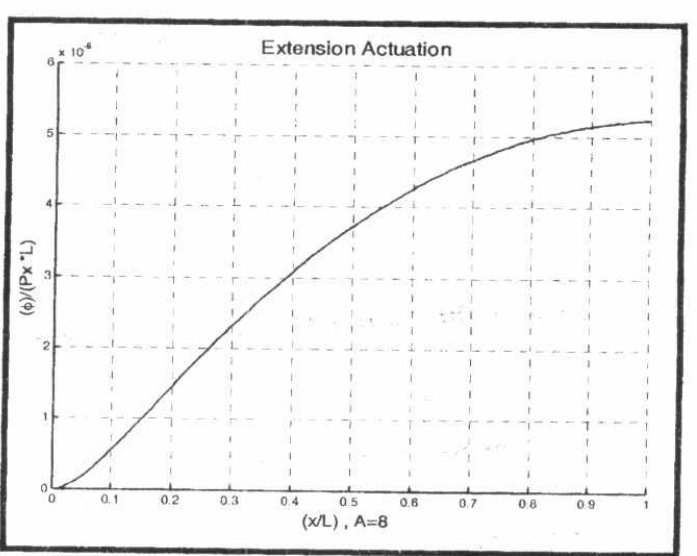

Fig. - 8 twist angle distribution for extension-twist actuation

Table 1 Mechanical Properties of materials

\begin{tabular}{|c|c|c|c|c|}
\hline \multicolumn{2}{|c|}{ Material Property } & $\begin{array}{c}\text { Glass/Epoxy } \\
\text { tape }\end{array}$ & PZT & PFC \\
\hline$E_{11}$ & $(\mathrm{MPa})$ & 38.40 & 42.06 & 62.74 \\
\hline$E_{22}$ & $(\mathrm{MPa})$ & 8.27 & 15.169 & 62.74 \\
\hline$v_{12}$ & & 0.26 & 0.25 & 0.30 \\
\hline$G_{12}$ & $(\mathrm{MPa})$ & 4.14 & 6.895 & 22.75 \\
\hline$d_{31}$ & $\mathrm{~cm} / \times 10^{-9}$ & 0 & -10.842 & -16.61 \\
\hline$d_{32}$ & $\mathrm{~cm} / 1^{-9}$ & 0 & -1.978 & -16.61 \\
\hline$\rho$ & $\mathrm{kg} / \mathrm{m}^{3}$ & 1800 & 5452.96 & 7944.16 \\
\hline$t$ & $\mathrm{~mm}$ & 0.125 & 0.102 & 0.2 \\
\hline
\end{tabular}


Table 2 - Average Twist Angle per unit load per unit length with changing aspect ratio

\begin{tabular}{|c|c|c|c|c|c|}
\hline \multicolumn{2}{|c|}{} & $(\mathrm{b} / \mathrm{a})=4$ & $(\mathrm{~b} / \mathrm{a})=6$ & $(\mathrm{~b} / \mathrm{a})=8$ & $(\mathrm{~b} / \mathrm{a})=10$ \\
\hline $\begin{array}{c}\text { Direct Twist } \\
\left(\mathrm{rad} / \mathrm{N} \mathrm{m}^{-2}\right)\end{array}$ & $\frac{\phi_{a v}}{m_{x} L}$ & 0.0019 & 0.0023 & 0.0026 & 0.0029 \\
\hline $\begin{array}{c}\text { Extension- } \\
\text { Coupling } \\
\left(\mathrm{rad} / \mathrm{N} \mathrm{m}^{-1}\right)\end{array}$ & $\frac{\phi_{a v}}{P_{x} L}$ & $5.62^{*} 10^{-7}$ & $7.99^{*} 10^{-7}$ & $9.83^{*} 10^{-7}$ & $1.13^{*} 10^{-6}$ \\
\hline $\begin{array}{c}\text { Warping-Beam1 } \\
\left(\mathrm{rad} / \mathrm{N} \mathrm{m}^{-3}\right)\end{array}$ & $\frac{\phi_{a v}}{B_{\omega} L}$ & $7.88^{*} 10^{-4}$ & 0.0012 & 0.0016 & 0.002 \\
\hline $\begin{array}{c}\text { Warping-Beam2 } \\
\left(\mathrm{rad} / \mathrm{N} \mathrm{m}^{-3}\right)\end{array}$ & $\frac{\phi_{a v}}{B_{\omega} L}$ & $7.88^{*} 10^{-4}$ & 0.0012 & 0.0016 & 0.002 \\
\hline $\begin{array}{c}\text { Warping-Beam3 } \\
\left(\mathrm{rad} / \mathrm{N} \mathrm{m}^{-3}\right)\end{array}$ & $\frac{\phi_{a v}}{B_{\omega} L}$ & 0.0114 & 0.0146 & 0.0173 & 0.0197 \\
\hline
\end{tabular}

Table 3-Average Twist Angle per unit load per unit length with changing plies number

\begin{tabular}{|c|c|c|c|c|c|}
\hline & & $\mathrm{pn}=8$ & $\mathrm{pn}=12$ & $\mathrm{pn}=16$ & $\mathrm{pn}=20$ \\
\hline $\begin{array}{c}\text { Direct Twist } \\
\left(\mathrm{rad} / \mathrm{N} \mathrm{m}^{-2}\right)\end{array}$ & $\frac{\phi_{a v}}{m_{x} L}$ & 0.0027 & 0.0027 & 0.0027 & 0.0027 \\
\hline $\begin{array}{c}\text { Extension- } \\
\text { Coupling } \\
\left(\mathrm{rad} / \mathrm{N} \mathrm{m}^{-1}\right)\end{array}$ & $\frac{\phi_{a v}}{P_{x} L}$ & $1.2 * 10^{-6}$ & $1.12^{*} 10^{-6}$ & $1.05^{*} 10^{-6}$ & $9.83^{*} 10^{-7}$ \\
\hline
\end{tabular}

Table 4-Average Twist Angle per unit load per unit length with changing beam length

\begin{tabular}{|c|c|c|c|c|}
\hline & $\mathrm{L}=300$ & $\mathrm{~L}=500$ & $\mathrm{~L}=700$ \\
\hline $\begin{array}{c}\text { Direct Twist } \\
\left(\mathrm{rad} / \mathrm{N} \mathrm{m}^{-2}\right)\end{array}$ & $\frac{\phi_{a v}}{m_{x} L}$ & 0.0137 & 0.0237 & 0.0337 \\
\hline $\begin{array}{c}\text { Extension- } \\
\text { Coupling } \\
\left(\mathrm{rad} / \mathrm{N} \mathrm{m}^{-1}\right)\end{array}$ & $\frac{\phi_{a v}}{P_{x} L}$ & $9.83^{*} 10^{-7}$ & $2.94^{*} 10^{-6}$ & $6.14^{*} 10^{-6}$ \\
\hline $\begin{array}{c}\text { Warping-Beam1 } \\
\left(\mathrm{rad} / \mathrm{N} \mathrm{m}^{-3}\right)\end{array}$ & $\frac{\phi_{a v}}{B_{w} L}$ & 0.0016 & 0.0010 & 0.0007 \\
\hline $\begin{array}{c}\text { Warping-Beam2 } \\
\left(\mathrm{rad} / \mathrm{N} \mathrm{m}^{-3}\right)\end{array}$ & $\frac{\phi_{a v}}{B_{w} L}$ & 0.0016 & 0.0010 & 0.0007 \\
\hline $\begin{array}{c}\text { Warping-Beam3 } \\
\left(\mathrm{rad} / \mathrm{N} \mathrm{m}^{-3}\right)\end{array}$ & $\frac{\phi_{a v}}{B_{w} L}$ & 0.0173 & 0.0180 & 0.0183 \\
\hline
\end{tabular}




\section{REFERENCES}

[1] A. H. Esmat, "Integrated Structural Tailoring and Control Using Smart Structures", Master thesis, MTC, Cairo 2001.

[2] R. Chandra, A. D. Stemple,I. Chopra,"Thin-walled Composite Beams Under Bending, Torsional, and Extensional loads", AIAA Journal Vol.28 No.7 1990.

[3] S. M. Ehlers, "Active Closed Cell Beam Shape Control", AIAA-94-1768-cp.

[4] C. Libove, "Stresses and Rate of Twist in Single-cell Thin-Walled Beams with Anisotropic Walls", AIAA Journal, Vol. 26, No. 9, 1988.

[5] M. Chaaban ,M. Elnomrossy, W.E. Abdel-Ghany, M. Kinawy, " Analytical Model of Active Thin-Walled Composite Beams under Bending Loading Introducing Wall-

Bending Effect", $8^{\text {th }}$ Conference of theoretical mechanics, Science Academy, Cairo, Egypt, 2005. 